\title{
Curriculum for Vestibular Medicine (VestMed) proposed by the Bárány Society
}

\section{The concept of Vestibular Medicine and the framework defining the knowledge, skills and attitudes needed for proficiency in Vestibular Medicine}

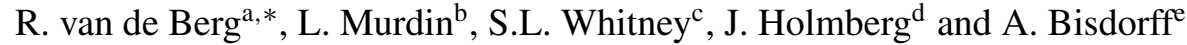 \\ ${ }^{a}$ Department of Otorhinolaryngology and Head and Neck Surgery, Maastricht University Medical Center, \\ School for Mental Health and Neuroscience, Maastricht, The Netherlands \\ ' Guy's and St Thomas' NHS Foundation Trust, and Ear Institute, UCL, London, UK \\ ${ }^{\mathrm{c}}$ Departments of Physical Therapy and Otolaryngology, University of Pittsburgh, Pittsburgh, PA, USA \\ ${ }^{\mathrm{d}}$ Intermountain Healthcare, Rehabilitation Services, Hearing and Balance Center, Salt Lake City, UT, USA \\ ${ }^{\mathrm{e}}$ Clinique du Vertige, Centre Hospitalier Emile Mayrisch, Esch-sur-Alzette, Luxembourg
}

\begin{abstract}
This document presents the initiative of the Bárány Society to improve diagnosis and care of patients presenting with vestibular symptoms worldwide.

The Vestibular Medicine (VestMed) concept embraces a wide approach to the potential causes of vestibular symptoms, acknowledging that vertigo, dizziness, and unsteadiness are non-specific symptoms that may arise from a broad spectrum of disorders, spanning from the inner ear to the brainstem, cerebellum and supratentorial cerebral networks, to many disorders beyond these structures.

The Bárány Society Vestibular Medicine Curriculum (BS-VestMed-Cur) is based on the concept that VestMed is practiced by different physician specialties and non-physician allied health professionals. Each profession has its characteristic disciplinary role and profile, but all work in overlapping areas. Each discipline requires good awareness of the variety of disorders that can present with vestibular symptoms, their underlying mechanisms and etiologies, diagnostic criteria and treatment options. Similarly, all disciplines require an understanding of their own limitations, the contribution to patient care from other professionals and when to involve other members of the VestMed community. Therefore, the BS-VestMed-Cur is the same for all health professionals involved, the overlaps and differences of the various relevant professions being defined by different levels of detail and depth of knowledge and skills.

The BS-VestMed-Cur defines a Basic and an Expert Level Curriculum. The Basic Level Curriculum covers the VestMed topics in less detail and depth, yet still conveys the concept of the wide net approach. It is designed for health professionals as an introduction to, and first step toward, VestMed expertise. The Expert Level Curriculum defines a Focused and Broad Expert. It covers the VestMed spectrum in high detail and requires a high level of understanding. In the Basic and Expert Level Curricula, the range of topics is the same and runs from anatomy, physiology and physics of the vestibular system, to vestibular symptoms, history taking, bedside examination, ancillary testing, the various vestibular disorders, their treatment and professional attitudes. Additionally, research topics relevant to clinical practice are included in the Expert Level Curriculum. For Focused Expert proficiency, the Basic Level Curriculum is required to ensure a broad overview and additionally requires an expansion of knowledge and skills in one or a few specific topics related to the focused expertise, e.g. inner ear surgery. Broad Expert proficiency targets professionals who deal with all sorts of patients presenting with vestibular symptoms (e.g. otorhinolaryngologists, neurologists, audiovestibular physicians, physical therapists), requiring a high level of VestMed expertise across the whole spectrum. For the Broad Expert, the Expert Level Curriculum is required in which the minimum attainment targets for all the topics go beyond the Basic Level Curriculum. The minimum requirements regarding knowledge and skills vary between Broad Experts, since they are tuned to the activity profile and underlying specialty of the expert.
\end{abstract}

\footnotetext{
*Corresponding author: Raymond van de Berg, Maastricht University Medical Center+ P. Debyelaan 25, 6229 HX Maastricht, The Netherlands. Tel.: +31 433874594/+31 654978648; E-mail:
}

raymond.vande.berg@mumc.nl.. ORCID: 0000-0001-9734-8668

ISSN 0957-4271 @ 2022 - The authors. Published by IOS Press. This is an Open Access article distributed under the terms of the Creative Commons Attribution-NonCommercial License (CC BY-NC 4.0). 
The BS-VestMed-Cur aims to provide a basis for current and future teaching and training programs for physicians and non-physicians. The Basic Level Curriculum could also serve as a resource for inspiration for teaching VestMed to students, postgraduate generalists such as primary care physicians and undergraduate health professionals, or anybody wishing to enter VestMed.

VestMed is considered a set of competences related to an area of practice of established physician specialties and nonphysician health professions rather than a separate clinical specialty. This curriculum does not aim to define a new single clinical specialty.

The BS-VestMed-Cur should also integrate with, facilitate and encourage translational research in the vestibular field.

\section{Introduction}

Vertigo, dizziness and unsteadiness are commonly encountered symptoms in clinical practice and emergency rooms, with an estimated lifetime prevalence of $17-30 \%$ [1-7]. They can result from peripheral and central as well as functional vestibular disorders, but also from other medical conditions. Vestibular disorders have a lifetime prevalence of up to $10 \%$ $[3,7,8]$. Despite this high prevalence, the attention they receive in healthcare falls far short of the need, with the consequence that up to $80 \%$ of patients are not correctly diagnosed or managed, depending on the clinical setting [1, 5, 6, 9-13]. Misdiagnoses can lead to unnecessary diagnostic testing, ineffective treatments [6], increased individual impairment and reduced quality of life, secondary functional disorders, $[14,15]$, chronicity and a high socio-economic burden [10,14-16]. It is therefore imperative that care for patients with vestibular disorders be improved.

Multiple factors contribute to the high prevalence of misdiagnoses and ineffective treatments of patients with vestibular disorders. They include inadequate coverage in undergraduate and postgraduate professional training curricula, non-existent standards in diagnostics and therapy, lack of normative values for laboratory tests, and the scarcity of translational research and randomized-controlled multicenter clinical trials in the vestibular field $[6,17]$. However, a proportion of the misdiagnosis and ineffective treatment is directly related to the limited knowledge, skills and attitudes of individual clinicians and therapists. Examples include: insufficient history taking and physical examination, misconceptions on how to interpret the findings of clinical examination and ancillary testing, lack of transdisciplinary cooperation $[6,12,18]$, unawareness of the range of differential diagnoses, and myths concerning the origins of vertigo (Seemungal et al., manuscript in preparation). All these factors highlight the need for a standardized, globally accepted, Vestibular Medicine curriculum on which to base the education and training of clinicians and therapists.
The development of a Vestibular Medicine curriculum must meet a range of challenges, as the vestibular system interacts with multiple other senses over many areas of the brain, with influences on spatial orientation, motor programming, autonomic function, emotion, and cognition. Because of the way healthcare is currently organized, disorders of this complex system cross the boundaries of various traditional clinical specialties (Table 1) $[5,6$, 19, 20]. Furthermore, the main vestibular symptoms (dizziness, vertigo and unsteadiness) can also be attributed to dysfunctions outside the vestibular system, e.g. cardiovascular, respiratory, psychiatric, or metabolic disorders, as well as to the unwanted side

Table 1

Examples of health professionals potentially involved in Vestibular Medicine

Physician specialists:

Neurologists*^

Otorhinolaryngologists $* \wedge$

Audiovestibular Physicians*

Ophthalmologists ${ }^{\wedge}$

Psychiatrists $^{\wedge}$

Emergency Medicine specialists ${ }^{\wedge}$

Rehabilitation Medicine specialists $\wedge$

Geriatricians $^{\circ}$

Pediatricians $^{\circ}$

General Internal Medicine specialists ${ }^{\circ}$

General practitioners ${ }^{\circ}$

Cardiologists $^{\wedge}$

Non-physician health professionals:

Physical Therapists*^

Audiologists*^

Physician Assistants $^{\wedge}$

Nurses and Nurse Practitioners ${ }^{\wedge}$

Laboratory Technicians $^{\wedge}$

Psychologists $^{\wedge}$

Orthopticians $^{\wedge}$

Behavioral Therapists $^{\wedge}$

Medical/Clinical/Technical physicists ${ }^{\wedge}$

Legend: (see "4.2.3 Three levels of VestMed proficiency”). ${ }^{\circ}=$ at least Basic proficiency recommended. * = mainly Broad Expert proficiency applicable. ${ }^{\wedge}=$ mainly Focused Expert proficiency applicable. ${ }^{* \wedge}=$ most likely both Broad and Focused Expert proficiency applicable, depending on the clinical practice of the professional. 
effects of medications, and to intoxications [21]. This implies that first the concept of Vestibular Medicine should be to explicitly specify the target population and the spectrum of disorders belonging to Vestibular Medicine. A Vestibular Medicine curriculum would then be able to encourage training and teaching initiatives within this field. Such a curriculum should be framed to accommodate the complexity of the topic, and to reflect the way physician specialties (British English: medical specialties) and non-physician health professions (British English: non-medical specialties) are organized.

There are many teaching and training programs for various related physician specialties (e.g. neurology and otorhinolaryngology, audiovestibular medicine (in some countries)) and non-physician health professions (e.g. physiotherapy, audiology, behavioral therapy) that diagnose and treat vestibular disorders [22]. These programs vary widely regarding content and they tend to reflect the scattered care of patients presenting with vestibular symptoms by focusing on a particular physician specialty or non-physician health profession and on topics considered relevant within that specialty or profession, neglecting or ignoring other aspects.

This document proposes two new entities: the concept of Vestibular Medicine (VestMed) and the consensus-based "Bárány Society Vestibular Medicine Curriculum" (BS-VestMed-Cur), to help improve standards of care for vestibular patients. The curriculum sets out a wide range of attainment targets while paying heed to the specific roles and profiles of the various physician specialties and non-physician health professions involved. It goes beyond current teaching and training initiatives and could therefore serve as a foundation for present and future education initiatives within the vestibular field.

\section{Methods}

\subsection{Development of the VestMed concept and the Bárány Society VestMed Curriculum}

The work presented here was initiated by members of the Classification Oversight Committee of the Bárány Society, set up to develop an International Classification of Vestibular Disorders (ICVD). The ICVD was founded in 2006 to establish evidencebased consensus and standardization regarding symptoms, signs, syndromes, diseases, diagnostic procedures, and treatments in the vestibular field [23-33].
Two of the authors (AB, RvdB) started by discussing and defining the goal, name and target population of the VestMed concept and the BS-VestMedCur. The same two authors then drafted a preliminary concept and BS-VestMed-Cur. The latter comprised two different curricula (Basic Level Curriculum and Expert Level Curriculum), including a framework with attainment targets regarding the knowledge, skills, and attitudes they considered relevant. The Neuro-otology curriculum of the American Academy of Neurology Neuro-ophthalmology/Neuro-otology Fellowship Core Curriculum [34] was used as a starting point, and topics regarding knowledge, skills and attitudes were added, removed, changed, and/or reorganized to comply with the objectives and layout of the BS-VestMed-Cur.

The draft curriculum and this accompanying article were the subject of an internal review process of the Bárány Society. This involved an iterative process of discussion and refinement, using online meetings and email exchanges. Comments were gathered from members of the Classification Committee, basic scientists, clinicians, and the Board of the Bárány Society. These comments were implemented where appropriate. The final version of the curriculum and this accompanying article were presented in September 2021 to the Board of the Bárány Society for tentative approval.

Disorders were included in the BS-VestMed-Cur if they could present with vertigo and/or dizziness and/or unsteadiness as one of the main symptoms. This inclusion criterion assumes that a clinician dealing with vestibular disorders can be part of the initial assessment of a patient with such a disorder. Subsequently, these disorders were classified into the three main vestibular syndromes: acute, episodic and chronic [21]. Disorders were categorized in the syndrome they were most likely to present with. Symptom presentation could fit into more than one syndrome type. Systemic disorders with vestibular manifestations that would fit variably into the three syndromes (e.g. auto-immune disorders) were categorized in a separate group.

The authors acknowledge that controversies and areas of disagreement exist in VestMed, e.g. how much neuro-ophthalmology should be part of the curriculum, the differences and overlaps of attainment levels for different professions, how to define the Basic Level and the concept of the Focused Expert, and the purposes the paper should serve. Each of these aspects was separately discussed between authors, to reach a final decision. 
For each aspect, consensus was reached between the authors.

Basic scientists, each of whom was a member of the Bárány Society, contributed to identifying the relevant research topics related to the various subject areas of the curriculum. A preliminary version of the Expert Curriculum including suggestions for relevant research topics was sent to the basic scientists. The suggested research topics were modified and new topics were added by them. This resulted in the final overview of research topics included in the Expert Curriculum.

The suggested minimum requirements in terms of knowledge and skills are graded according to the system used in the UEMS European Training Requirements for Neurology [35]. This system was chosen to reflect, to a certain extent, "Miller's prism of clinical competence" [36]. It comprises different gradings for knowledge and skills:

Levels of knowledge in BS-VestMed-Cur:

1. Knows of

2. Knows basic concepts

3. Knows generally (able to make a complete diagnosis)

4. Knows specifically and broadly (competent to treat as far as possible), knowledge of own capacities and limitations, ready for referral to other specialists.

Levels of skills in BS-VestMed-Cur:

1. Has observed or knows of

2. Can manage with assistance

3. Can manage on the whole but may need assistance

4. Competent to manage without assistance including complications, knowledge of own capacities and limitations, ready for referral to other specialists.

Additionally, three abbreviations were used on indication:

1. NA: not applicable

2. NG: no grading

3. NRBLC: not required for Basic Level Curriculum

"Not applicable" is used in the case where knowledge and/or skills are not applicable for a certain topic. "No Grading" is used when grading knowledge and skills regarding treatment of some vestibular disorders (e.g. Mal de Débarquement syn- drome) is presently considered inappropriate. The consensus is that for some disorders, general information, advice or tentative treatments are available, but too little evidence or generally accepted expert opinion are currently established to recommend specific treatment options. "Not required for Basic Level Curriculum" is used for knowledge and skills relevant for certain health professions in the Expert Level Curriculum, but which are not required for the Basic Level Curriculum.

For both the Basic Level and Expert Level Curricula, the suggested minimum level required for each knowledge topic and skill was determined by Bárány Society members representing the various medical specialties and professions. The aim was to determine realistic levels, which should be attainable by many health professionals involved in the care of patients with vestibular disorders. Therefore, the indicated grades are minimal requirements suggested for health professionals wishing to be knowledgeable in VestMed (Basic Level Curriculum) or wishing to increase their proficiency beyond the basic Basic Level Curriculum (Expert Level Curriculum). Indicated grades do not reflect minimal requirements for the physician or non-physician specialty in general, e.g. the demands for middle ear anatomy for a middle ear surgeon would be higher than for an otolaryngologist with a focus on VestMed, as would the demands in neuro-vascular anatomy for a neurologist involved in interventional stroke care. Professional attitudes and research topics were not graded. It was decided that these attitudes should be fully present in all cases (Basic and Expert Level Curriculum). A general awareness of relevant research topics is required to be considered proficient in Vestibular Medicine at the Expert Level.

\subsection{Defining terminology}

During development of the VestMed concept and the BS-VestMed-Cur curriculum, it was found that terminology differed among countries and among clinical backgrounds. In consensus, the following terminology was defined and used for the development of the VestMed concept and BS-VestMed-Cur curriculum:

Vestibular symptoms: dizziness, vertigo, postural symptoms, vestibulo-visual symptoms [26]

Vestibular system: the vestibular part of the inner ear with its widespread connections to the infra- and supratentorial parts of the brain and its interaction 
with visual and proprioceptive afferents

Vestibular disorders: the wide spectrum of all disorders affecting vestibular function, including inner ear, vestibular nerves, and brain

Vestibular Medicine: all healthcare activities related to vestibular disorders

Physician specialty: medical specialty (surgical and non-surgical)

VestMed professional: physician specialists and non-physician health professionals practicing Vestibular Medicine

\section{The concept of Vestibular Medicine}

\subsection{Nomenclature and the spectrum of Vestibular Medicine (VestMed)}

Traditionally, clinical specialties focusing on the inner ear and its connections in the brain deal with the symptoms of vertigo, dizziness, and unsteadiness. Depending on the geographical region, terms like oto-neurology, neuro-otology or audiovestibular medicine are used. However, our understanding of the complexity of disorders presenting with vestibular symptoms has evolved in recent decades [19, 24-31]. As described above, vestibular symptoms are nonspecific symptoms which might indicate an inner ear, brainstem, or cerebellar disease. They may also indicate diseases beyond these structures [21]. The term "Vestibular Medicine" (VestMed) aims to reflect this wide spectrum of disorders, which is considered a more appropriate response to diagnosing and treating patients presenting with vestibular symptoms. VestMed explicitly goes beyond the traditional narrow perspective of inner ear, brainstem, or cerebellar disorders. Clinicians dealing with vestibular symptoms therefore need to expand their knowledge, skills and attitudes beyond oto-neurology. Proficiency in VestMed requires a core set of knowledge, skills and attitudes and a good awareness of the wide spectrum of the topic.

\subsection{VestMed is an added competence to established physician specialties and non-physician health professions}

The Bárány Society's position is that no disease or disorder or syndrome or method belongs exclusively to a particular physician specialty or non-physician health profession. VestMed therefore encompasses all professionals potentially involved in diagnosing and treating patients with vestibular disorders (examples illustrated in Table 1), and seeking to develop their expertise. Therefore, VestMed should be considered as an intrinsic or additional extended competence to established physician specialties and non-physician health professions. It aims to be as inclusive as possible: physician specialties and non-physician health professions not mentioned in Table 1 are welcome to be included in the future, should they express that wish. VestMed is explicitly not meant to develop into a separate single clinical specialty, since there is little likelihood of one specialist embracing at a high level the full range of knowledge and skills required to diagnose and treat the wide spectrum of clinical conditions presenting with vestibular symptoms. In other words, multidisciplinary working is currently and is anticipated to remain essential to good practice in VestMed. Also, a VestMed professional should understand the technological and physical principles and limitations of diagnostic equipment and procedures. These factors should facilitate constructive interdisciplinary discussions, and hence improve vestibular care [18]. The Bárány Society is mindful that all health professionals dealing with patients with vestibular disorders should share a common core of knowledge, skills and attitudes, since patients might present with the same disorder to VestMed professionals with different clinical backgrounds. The boundaries and overlaps between different clinical backgrounds may vary according to local medical culture, traditions, and healthcare settings.

\section{The Bárány Society Vestibular Medicine Curriculum}

\subsection{Goal of the BS-VestMed-Cur}

BS-VestMed-Cur sets out a comprehensive framework of suggested topics and skills, with attainment targets required for a VestMed professional, at a basic level (Basic Level Curriculum) and expert level (Expert Level Curriculum). All professionals should have high standards in the areas of Vestibular Medicine closely related to their physician specialty or non-physician health profession, but they should also be aware of areas beyond their own specialty or profession. Professional attitudes include an understanding of one's own limitations and how to relate to professionals with VestMed expertise beyond one's own core competence. Ideally, VestMed 
professionals practice in a team or network covering the whole spectrum, with all members knowing their core competence and limitations, and when and how they relate or refer to other members of the team. The Bárány Society, with its broad base of members involved in clinical, basic, and translational research, believes that anybody claiming to be highly proficient in VestMed should be aware of the ongoing research in this field. In the Expert Level Curriculum, the curriculum consequently incorporates items on research topics and methods, an awareness of which is considered fundamental for a deeper understanding of VestMed.

By defining standards necessary to become proficient in VestMed, and by providing a foundation for teaching and training initiatives within the vestibular field, the eventual goal of the BS-VestMed-Cur is to improve clinical care for patients with vestibular disorders worldwide and to encourage translational research.

\subsection{Outline of the BS-VestMed-Cur}

The curriculum describes all the areas required for sound competence in VestMed. It sets out a list of the relevant topics but does not discuss them in textbook detail. Two curricula are included: The Basic Level Curriculum and the Expert Level Curriculum.

\subsubsection{The Basic Level Curriculum}

Tables $1 \mathrm{a}-1 \mathrm{~h}$ in the Supplementary materials present the Basic Level Curriculum. It is designed for health professionals as an introduction to, and first step toward, VestMed expertise. This curriculum therefore covers the whole range of VestMed topics with limited detail and at a basic level of depth. Subject areas include: Anatomy, Physiology \& Physics; Vestibular symptoms; History taking; Bedside examination; Ancillary testing; Disorders; Treatment; Professional Attitudes. This reflects a broad-brush approach to reflect the wide variety of vestibular disorders and the many conditions that may present with prominent vestibular symptoms such as vertigo, dizziness and unsteadiness as defined above. The main goal is to ensure that VestMed professionals acquire this broad overview. In the Basic Level Curriculum, the minimum requirements regarding knowledge topics and skills are graded the same for all health professions. Since this curriculum requires only a basic level of VestMed understanding, many topics have lower minimum requirements than the Expert Level Curriculum and the focus is mainly on the knowledge topics and attitudes and less on the skills.

\subsubsection{The Expert Level Curriculum}

Tables $2 \mathrm{a}-2 \mathrm{i}$ in the Supplementary materials present the Expert Level Curriculum. This curriculum is designed for health professionals wishing to increase their VestMed proficiency to expert level. Anyone practicing VestMed as a main or important focus should master the wide range of knowledge topics and skills at a high level. The Expert Level Curriculum therefore covers the whole VestMed spectrum in high detail and a high level of understanding is required. The same subject areas are included as in the Basic Level Curriculum (Anatomy, Physiology \& Physics; Vestibular symptoms; etc.), and the subject area "Research topics" is added. However, subject areas in the Expert Level Curriculum are much more detailed than in the Basic Level Curriculum. For example, the Expert Level Curriculum includes much more detail on differential diagnoses of vestibular syndromes and types of nystagmus.

Since the Expert Level Curriculum aims at a high proficiency in VestMed, the grading regarding minimum requirements of understanding is usually higher than in the Basic Level Curriculum. Importantly, grading in the Expert Level Curriculum differs among VestMed professionals with different clinical backgrounds. Attainment targets are specified for each physician specialty and non-physician health profession involved in the care of patients with vestibular disorders, to reflect their respective activity profile and expected scope of practice. For example, it is suggested that an otorhinolaryngologist highly proficient in VestMed should be able to treat vestibular migraine, while VestMed professionals with many different clinical backgrounds should be able to treat BPPV. However, a VestMed professional with a background in neurology, audiovestibular medicine or physiotherapy will not be expected to perform surgery, but should have a basic understanding of the available procedures and when to refer for consideration of these. Thus, the curriculum for each physician specialty and non-physician health profession is the same but the suggested minimum requirements for knowledge and skills will vary. As a result, each clinical specialty has its own particular BS-VestMed-Cur profile.

Differences between the Basic Level Curriculum and the Expert Level Curriculum are presented in Table 2. 
Table 2

Differences between the Basic Level Curriculum and Expert Level Curriculum

\begin{tabular}{lccl}
\hline Level & Subject areas & $\begin{array}{l}\text { Detail within } \\
\text { subject areas }\end{array}$ & $\begin{array}{l}\text { Level of knowledge } \\
\text { and skills }\end{array}$ \\
\hline Basic & 8 & limited & $\begin{array}{l}\text { - Basic minimum requirements } \\
- \text { All clinical backgrounds have the same grading }\end{array}$ \\
\hline Expert & $\begin{array}{c}8+\text { Research } \\
\text { topics }\end{array}$ & high & $\begin{array}{l}\text { - High minimum requirements } \\
- \text { Each clinical background has } \\
\text { its own grading (VestMed profiles) }\end{array}$ \\
\hline
\end{tabular}

\subsubsection{Three levels of VestMed proficiency}

The BS-VestMed-Cur consists of a Basic and Expert Level Curriculum, since this facilitates the definition of three different levels of VestMed proficiency, which are relevant for clinical practice: Basic proficiency, Broad Expert proficiency and Focused Expert proficiency. This is illustrated in Table 3.

The Basic Level Curriculum is considered the basis for all VestMed professionals. Its underpinning approach is intended to promote a low-threshold access to the fundamentals of VestMed and thus to encourage many professionals to become part of the VestMed community at large. Mastering the knowledge and skills of the Basic level curriculum leads to Basic proficiency in VestMed. VestMed professionals should obtain a higher proficiency than described in the Basic Level Curriculum. This can be achieved with the more detailed Expert Level Curriculum (Tables 2 and 3). If health professionals desire to practice VestMed as a main activity within their own clinical background, it would be preferable to master the whole Expert Level Curriculum at the levels defined for their specific clinical backgrounds. This leads to Broad Expert proficiency in VestMed. However, this does not imply that each VestMed professional needs to master the whole VestMed spectrum as described in the Expert Level Curriculum. For example, a cochlear implant surgeon might benefit from additional knowledge and skills from the Expert Level Curriculum aimed at diagnosing and treating peripheral vestibular hypofunction but does not need specific knowledge to recognize certain cerebellar disorders. Therefore, the Expert Level Curriculum can also be used to increase proficiency on certain topics, tailored to the clinical practice of each VestMed professional. A Focused Expert proficiency in VestMed is attained via an expansion in detail and depth of a limited number of topics of the Basic Level Curriculum to expert levels. Future training and teaching programs might define which topics of the Expert Level Curriculum are relevant to significantly increase Focused Expert proficiency in defined health professionals.

Table 1 indicates for clinical specialties and health professions whether Basic, Broad Expert, Focused Expert, or Broad and Focused Expert proficiency is most likely to be applicable.

\subsubsection{Rationale behind topics included in the BS-VestMed-Cur}

The BS-VestMed-Cur spectrum ranges from basic science VestMed topics (e.g. the anatomy of the vestibular system), to rare conditions with vestibular presentations, and highly selective treatment procedures that are only performed in tertiary referral centres. Even so, the curriculum probably does not

Table 3

Three levels of VestMed proficiency

\begin{tabular}{ll}
\hline Level of proficiency & $\begin{array}{l}\text { BS-VestMed-Cur curricula } \\
\text { needed to reach proficiency }\end{array}$ \\
\hline Basic & Basic Level Curriculum \\
Broad Expert & Expert Level Curriculum \\
Focused Expert & Basic Level Curriculum + \\
& only specific topics of Expert Level Curriculum \\
\hline
\end{tabular}

Legend: Three levels of VestMed proficiency and an overview of the BS-VestMed-Cur curricula needed to reach these levels. To reach Basic proficiency, mastering the topics of the Basic Level Curriculum is sufficient. To reach Broad Expert proficiency, mastering both the Basic Level and Expert Level curriculum is necessary. It should be noted that the required level of knowledge and skills differs between professions in the Expert Level Curriculum. Focused Expert proficiency can be attained by understanding the Basic Level Curriculum, complemented with specific topics of the Expert Level Curriculum, tailored to the clinical practice of the VestMed professional. 
capture all possible disorders presenting with vestibular symptoms. However, the consensus was that the topics incorporated in BS-VestMed-Cur represent the minimum requirements for knowledge, skills, and attitudes needed to become proficient in VestMed. The Basic Level Curriculum is also recommended for basic scientists as a resource for keeping updated with the clinical VestMed spectrum.

\subsubsection{Rationale behind grading of knowledge and skills in the BS-VestMed-Cur}

The BS-VestMed-Cur differentiates between knowledge, skills, and attitudes. Knowledge and skills are graded according to the system used in the UEMS European Training Requirements for Neurology [35].

Grading in the Basic Level Curriculum does not differentiate between clinical backgrounds. This implies that it is designed for any health professional wishing to become proficient in VestMed, regardless of clinical background. The Expert Level Curriculum currently, only proposes grading for neurology, otorhinolaryngology, audiovestibular medicine and physiotherapy. Expansion to other physician specialties and non-physician health professions is expected to follow in the future in cooperation with the Bárány Society. Regarding audiovestibular medicine, multiple clinical backgrounds are possible for professionals practicing this specialty. Grading in this curriculum reflects the minimum requirements for physicians who followed specialist training in audiovestibular medicine (e.g. in the United Kingdom where this is a recognized physician subspecialty).

Professional attitudes and research topics are not graded, since the consensus was that these attitudes should be fully present in VestMed professionals at both Basic and Expert Levels. A general awareness of research topics is required for the Expert Level Curriculum.

The BS-VestMed-Cur grades knowledge and skills separately. For some topics, the difference between knowledge and skills is quite clear, e.g. knowing the "vestibular symptoms" is considered knowledge, while "being able to extract vestibular symptoms when taking the history" is considered a skill. However, the authors are aware that knowledge and skills are often interrelated and that a separate grading might be artificial to some extent. Generally speaking, "Knowledge" refers to understanding around mechanisms of disease, diagnostic processes and principles of management. "Skills" refers to more practical aspects of care.
In relation to "Disorders and diseases", "knowledge" and "skills" were considered as equivalent and so indivisible; hence there are no "skills" defined for "Disorders and diseases".

In relation to "Bedside examination", "skill" is the ability to execute the examination practically, and "knowledge" is the ability to interpret the observed findings.

In relation to "Treatment", "knowledge" relates to understanding of the theoretical principles, whereas "skills" refers to the ability to deliver the treatment under consideration. This distinction shows that clinical professionals may be aware of a treatment that a patient may need ("knowledge"), whilst lacking the "skills" to deliver it themselves personally. In this case, it is the "knowledge" element that allows appropriate onwards referral to be made, showing that "knowledge" can be important independently of "skills".

The "skills" section gradings refer to the minimum required level necessary for each physician specialty and non-physician health profession, but the highest level (level 4) is required where clinicians are taking overall independent responsibility for management of vestibular conditions.

It is also emphasised that the BS-VestMed-Cur topics and their grading are not "set in stone": they are open for review in relation to future developments in VestMed.

\section{Implementation and future perspectives}

BS-VestMed-Cur does not go into textbook levels of detail nor does it define the design of a VestMed training program or criteria for trainers. For future implementation of the curriculum, multiple aspects could be considered. First, the curriculum should be a guide for institutions and individuals involved in teaching Vestibular Medicine, to make sure that their programs cover all the relevant topics. The hope is that the framework provided in this curriculum will assist those seeking to raise standards of content and delivery for current and future teaching and training programs, resulting in well-organized teaching materials and training centers, and culminating in certification for added competencies.

As stated above, the Basic Level Curriculum is the basis for all VestMed professionals but the degree to which the Expert Level Curriculum needs to be mastered depends on the health care setting, leading to Broad Expert or Focused Expert proficiency 
in VestMed. Regarding Focused Expert proficiency, teaching and training programs are encouraged to define which topics of the Expert Level Curriculum are relevant. It might be hypothesized that "submodules" could be defined within the Expert Level Curriculum that might be relevant for specific populations of VestMed professionals to reach the level of Focused Expert proficiency required within their clinical specialty or profession, yet never neglecting the Basic Curriculum.

Physician specialties and non-physician health professions not yet included in this curriculum are invited to become part of BS-VestMed-Cur. The Bárány Society will establish an Educational Committee to promote the curriculum and to facilitate its implementation. This committee will also have the responsibility for keeping BS-VestMed-Cur up-todate through periodic review. BS-VestMed-Cur will need regular updating depending on clinical and scientific developments, new insights, and the needs of the learners. The Bárány Society will try to cooperate with other societies and training programs to identify relevant teaching materials and highlight any gaps in the existing available provision. This could facilitate the development of a complete set of teaching and training materials that covers all VestMed topics at the suggested minimum requirements and beyond. Furthermore, the possibility of establishing accredited VestMed masterclasses and VestMed training programs for different clinical backgrounds will be explored. All these efforts might eventually result in an online hub of evidence-based teaching materials customized to the level of knowledge, skills, and attitudes of each clinical specialty and profession, and facilitate the development of designated expert training centers, masterclasses, and training programs. Finally, the developers of BS-VestMedCur also hope that this curriculum will cross-fertilize clinical practice and research in the vestibular field.

In summary, the BS-VestMed-Cur intends to improve the care of patients with vestibular disorders by promoting high quality vestibular training worldwide for all professionals involved with patients with vestibular disorders and with translational research in this field.

\section{Acknowledgments}

With helpful comments from Michael Strupp, Thomas Lempert, Joseph Furman, Herman Kingma, Angelica Perez-Fornos, Christophe Lopez, Michael
Schubert, Jeffrey Staab, Måns Magnusson and Göran Laurell.

\section{Supplementary material}

The supplementary material is available in the electronic version of this article: https://dx.doi.org/ 10.3233/VES-210095.

\section{References}

[1] K. Feil, R. Feuerecker, N. Goldschagg, R. Strobl, T. Brandt, A. von Muller, et al., Predictive Capability of an iPad-Based Medical Device (medx) for the Diagnosis of Vertigo and Dizziness, Front Neurol 9 (2018), 29.

[2] B. Gopinath, C.M. McMahon, E. Rochtchina and P. Mitchell, Dizziness and vertigo in an older population: the Blue Mountains prospective cross-sectional study, Clin Otolaryngol 34(6) (2009), 552-556.

[3] E. Grill, M. Heuberger, R. Strobl, M. Saglam, R. Holle, B. Linkohr, et al., Prevalence, Determinants, and Consequences of Vestibular Hypofunction. Results From the KORA-FF4 Survey, Front Neurol 9 (2018), 1076.

[4] E. Kovacs, X. Wang and E. Grill, Economic burden of vertigo: a systematic review, Health Econ Rev 9(1) (2019), 37.

[5] R. van de Berg, M. van Tilburg and H. Kingma, Bilateral Vestibular Hypofunction: Challenges in Establishing the Diagnosis in Adults, ORL J Otorhinolaryngol Relat Spec 77(4) (2015), 197-218.

[6] A. Zwergal, T. Brandt, M. Magnusson and C. Kennard, DIZZYNET-a European network initiative for vertigo and balance research: visions and aims, J Neurol 263(Suppl 1) (2016), S2-9.

[7] L. Murdin and A.G. Schilder, Epidemiology of balance symptoms and disorders in the community: a systematic review, Otol Neurotol 36(3) (2015), 387-392.

[8] H.K. Neuhauser, The epidemiology of dizziness and vertigo, Handb Clin Neurol 137 (2016), 67-82.

[9] R. Geser and D. Straumann, Referral and final diagnoses of patients assessed in an academic vertigo center, Front Neurol 3 (2012), 169.

[10] E. Grill, M. Strupp, M. Muller and K. Jahn, Health services utilization of patients with vertigo in primary care: a retrospective cohort study, J Neurol 261(8) (2014), 1492-1498.

[11] K.A. Kerber, L.B. Morgenstern, W.J. Meurer, T. McLaughlin, P.A. Hall, J. Forman, et al., Nystagmus assessments documented by emergency physicians in acute dizziness presentations: a target for decision support? Acad Emerg Med 18(6) (2011), 619-626.

[12] K.A. Kerber and D.E. Newman-Toker, Misdiagnosing Dizzy Patients: Common Pitfalls in Clinical Practice, Neurol Clin 33(3) (2015), 565-575, viii.

[13] G. Royl, C.J. Ploner and C. Leithner, Dizziness in the emergency room: diagnoses and misdiagnoses, Eur Neurol 66(5) (2011), 256-263.

[14] H.K. Neuhauser, A. Radtke, M. von Brevern, F. Lezius, M. Feldmann and T. Lempert, Burden of dizziness and vertigo in the community, Arch Intern Med 168(19) (2008), 2118-2124. 
[15] H.K. Neuhauser, M. von Brevern, A. Radtke, F. Lezius, M. Feldmann, T. Ziese, et al., Epidemiology of vestibular vertigo: a neurotologic survey of the general population, Neurology 65(6) (2005), 898-904.

[16] A.S. Saber Tehrani, D. Coughlan, Y.H. Hsieh, G. Mantokoudis, F.K. Korley, K.A. Kerber, et al., Rising annual costs of dizziness presentations to U.S. emergency departments, Acad Emerg Med 20(7) (2013), 689-696.

[17] M. Strupp, J. Grimberg, J. Teufel, G. Laurell, H. Kingma and E. Grill, Worldwide survey on laboratory testing of vestibular function, Neurology: Clinical Practice (2019), 10.1212/CPJ.0000000000000744.

[18] P. Gerretsen, P. Shah, A. Logotheti, M. Attia, T. Balakumar, S. Sulway, et al., Interdisciplinary integration of nursing and psychiatry (INaP) improves dizziness-related disability, Laryngoscope 130(7) (2020), 1800-1804.

[19] A. Bisdorff, Vestibular symptoms and history taking, Handb Clin Neurol 137 (2016), 83-90.

[20] H. Kingma, R. van de Berg, Anatomy, physiology, and physics of the peripheral vestibular system, Handb Clin Neurol 137 (2016), 1-16.

[21] D.E. Newman-Toker and J.A. Edlow, TiTrATE: A Novel, Evidence-Based Approach to Diagnosing Acute Dizziness and Vertigo, Neurol Clin 33(3) (2015), 577-599, viii.

[22] H.S. Cohen, K.R. Gottshall, M. Graziano, E.M. Malmstrom, M.H. Sharpe, S.L. Whitney, et al., International guidelines for education in vestibular rehabilitation therapy, $J$ Vestib Res 21(5) (2011), 243-250.

[23] www.thebaranysociety.org

[24] Y. Agrawal, R. Van de Berg, F. Wuyts, L. Walther, M. Magnusson, E. Oh, et al., Presbyvestibulopathy: Diagnostic criteria Consensus document of the classification committee of the Barany Society, J Vestib Res 29(4) (2019), 161-170.

[25] A. Bisdorff, [The Barany Classification of vestibular disorders, its clinical implementation and future prospects], HNO 68(5) (2020), 304-312.

[26] A. Bisdorff, M. Von Brevern, T. Lempert and D.E. NewmanToker, Classification of vestibular symptoms: towards an international classification of vestibular disorders, $J$ Vestib Res 19(1-2) (2009), 1-13.

[27] S.D.Z. Eggers, A. Bisdorf, M. von Brevern, D.S. Zee, J.S. Kim, N. Perez-Fernandez, et al., Classification of vestibular signs and examination techniques: Nystagmus and nystagmus-like movements, J Vestib Res 29(2-3) (2019), $57-87$.
[28] J.A. Lopez-Escamez, J. Carey, W.H. Chung, J.A. Goebel, M. Magnusson, M. Mandala, et al., [Diagnostic criteria for Meniere's disease according to the Classification Committee of the Barany Society], HNO 65(11) (2017), 887-893.

[29] J.P. Staab, A. Eckhardt-Henn, A. Horii, R. Jacob, M. Strupp, T. Brandt, et al., Diagnostic criteria for persistent posturalperceptual dizziness (PPPD): Consensus document of the committee for the Classification of Vestibular Disorders of the Barany Society, J Vestib Res 27(4) (2017), 191-208.

[30] M. Strupp, J.S. Kim, T. Murofushi, D. Straumann, J.C. Jen, S.M. Rosengren, et al., Bilateral vestibulopathy: Diagnostic criteria Consensus document of the Classification Committee of the Barany Society, J Vestib Res 27(4) (2017), 177-189.

[31] M. von Brevern, P. Bertholon, T. Brandt, T. Fife, T. Imai, D. Nuti, et al., Benign paroxysmal positional vertigo: Diagnostic criteria Consensus document of the Committee for the Classification of Vestibular Disorders of the Barany Society, Acta Otorrinolaringol Esp 68(6) (2017), 349-360.

[32] M. Strupp, J.A. Lopez-Escamez, J.S. Kim, D. Straumann, J.C. Jen, J. Carey, et al., Vestibular paroxysmia: Diagnostic criteria, J Vestib Res 26(5-6) (2016), 409-415.

[33] A.R. Bisdorff, J.P. Staab and D.E. Newman-Toker, Overview of the International Classification of Vestibular Disorders, Neurol Clin 33(3) (2015), 541-550, vii.

[34] American Academy of Neurology Neuro-Ophthalmology/ Neuro-Otology Fellowship Core Curriculum [Available from: https://eur01.safelinks.protection.outlook.com/?url= https $\% 3 \mathrm{~A} \% 2 \mathrm{~F} \% 2 \mathrm{Fwww}$.aan.com\%2Fsiteassets\%2Fhomepage $\% 2$ Ftools-and-resources $\% 2$ Facademic-neurologist-re searchers $\% 2$ Fteaching-materials $\% 2$ Faan-core-curriculafor-program-directorstor\%2Fneuroophthalmology-fellows hip-core-curricula_tr.pdf \&data $=04 \% 7 \mathrm{C} 01 \% 7 \mathrm{Craymond}$. vande.berg\%40mumc.nl\%7C42540c25139646e49e5408d87 d7f3500\%7C08273b66221040cd8b205cf6cddc279a\%7C0 \%7C1\%7C637397332598257541\%7CUnknown\%7CTW FpbGZsb3d8eyJWIjoiMC4wLjAwMDAiLCJQIjoiV2lu MzIiLCJBTiI6Ik1haWwiLCJXVCI6Mn0\%3D\%7C1000 \&sdata=oa1FuMdhWIvFgWkxR9XuXS0Qgnv2ZAnGI4B jhiYWII0\%3D\&reserved=0.

[35] https://www.uems-neuroboard.org/web/images/docs/exam /European-Training-Requirements-Neurology-acceptedversion-21Oct16.pdf[

[36] G.E. Miller, The assessment of clinical skills/comp etence/performance, Acad Med 65(9 Suppl) (1990), S63-7. 